\title{
Corrigendum to "Hepatoprotective Role of Ethanolic Extract of Vitex negundo in Thioacetamide-Induced Liver Fibrosis in Male Rats"
}

\author{
Farkaad A. Kadir $\mathbb{D}^{1}{ }^{1}$ Normadiah M. Kassim $\mathbb{D}^{1}{ }^{1}$ \\ Mahmood A. Abdulla $\mathbb{1}^{2}{ }^{2}$ and Wageeh A. Yehye ${ }^{3}$ \\ ${ }^{1}$ Department of Anatomy, Faculty of Medicine, University of Malaya, 50603 Kuala Lumpur, Federal Territory, Malaysia \\ ${ }^{2}$ Department of Biomedical Science, Faculty of Medicine, University of Malaya, 50603 Kuala Lumpur, Federal Territory, Malaysia \\ ${ }^{3}$ Nanotechnology \& Catalysis Research Centre (NANOCAT), Block 3A, Institute of Postgraduate Studies Building, \\ University of Malaya, 50603 Kuala Lumpur, Federal Territory, Malaysia
}

Correspondence should be addressed to Normadiah M. Kassim; normadiah_mk@um.edu.my

Received 15 October 2018; Accepted 16 October 2018; Published 5 November 2018

Copyright (c) 2018 Farkaad A. Kadir et al. This is an open access article distributed under the Creative Commons Attribution License, which permits unrestricted use, distribution, and reproduction in any medium, provided the original work is properly cited.

In the article titled "Hepatoprotective Role of Ethanolic Extract of Vitex negundo in Thioacetamide-Induced Liver Fibrosis in Male Rats" [1], there was figure duplication in Figure 1, which showed the appearance of livers. Figure 1(b) is the same as Figure 1, TAA, in another article by the same group, Amin et al. [2], and Figures $1(\mathrm{f})$ and $1(\mathrm{~g})$ in [1] are the same.

An institutional investigation by the University of Malaya found there was no system to index and file data and images to avoid mislabeling and mishandling, which led to errors and duplication of research data. The authors did not thoroughly check the manuscript before submission.

Figures 1(b) and 1(f) were uploaded at revision after a reviewer asked for higher quality images. These images were selected in error by the authors. Moreover, Figures 1(c), 1(f), and $1(\mathrm{~g})$ are replaced by new images as the authors informed us that they had taken images at the end of the experiment. Accordingly, they selected new images to replace the previous images in these panels. The corrected Figure 1 is shown below. 


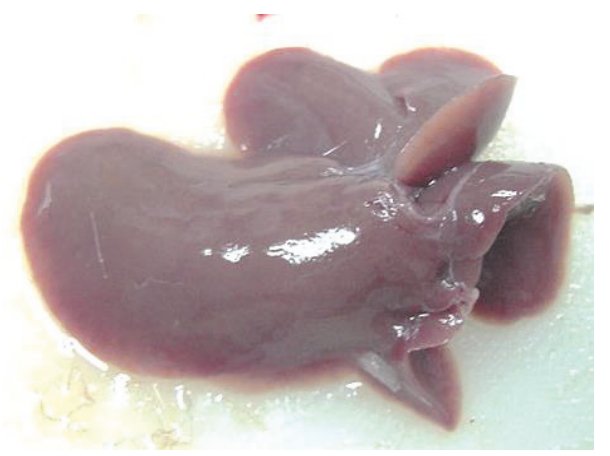

(a)

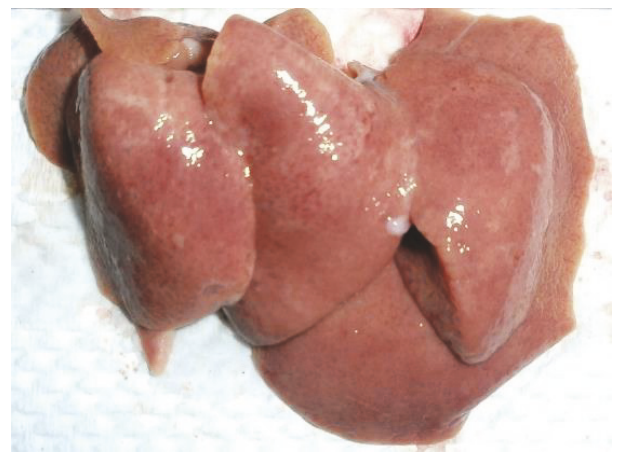

(c)

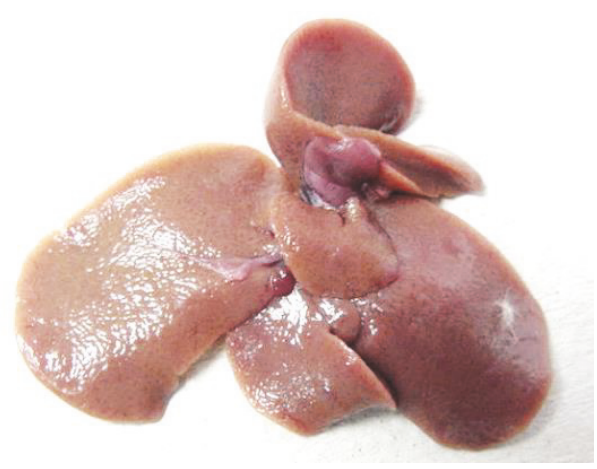

(e)

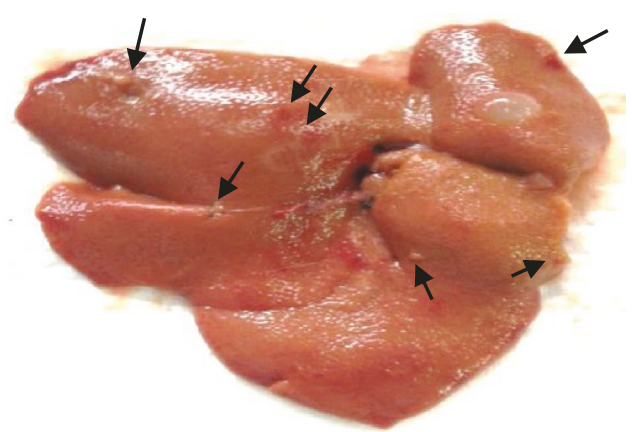

(b)

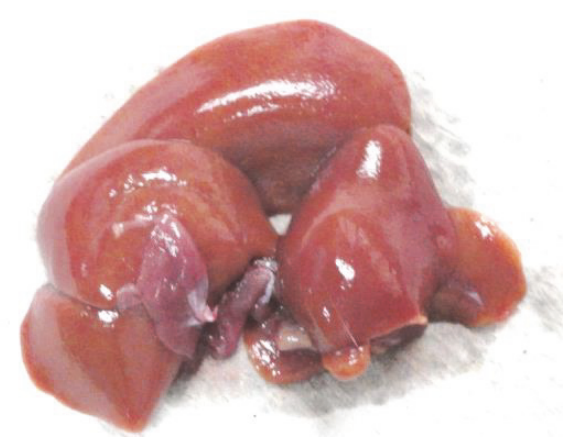

(d)

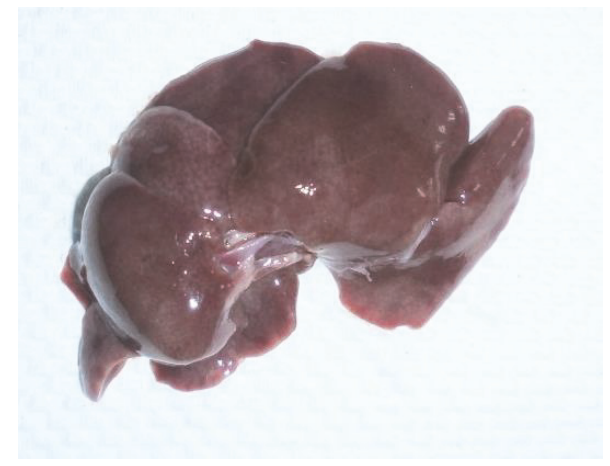

(f)

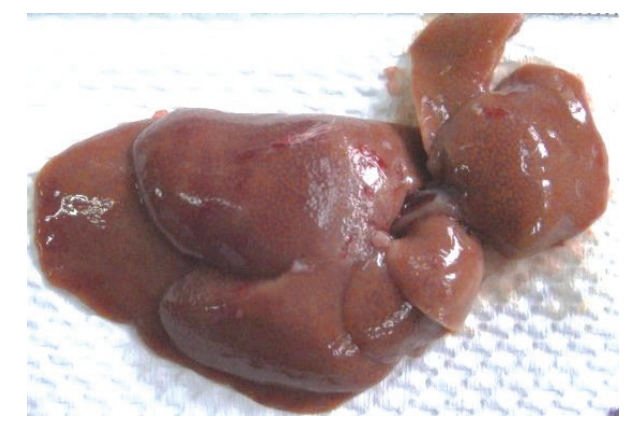

(g)

FIgURE 1: Photographs showing the macroscopic appearances of livers from different experimental groups: (a) control group-showing regular smooth surface, (b) TAA group (hepatotoxic group) - showing rough surface with micronodular distribution (black arrows), (c) SY + TAA group - showing smooth surface, (d) VN 100 group—showing liver with a smooth surface, (e) VN $100+$ TAA group-showing liver with a nearly smooth surface, (f) VN 300 group—showing liver with a smooth surface, and (g) VN $300+$ TAA group-showing liver with a smooth surface. 


\section{References}

[1] F. A. Kadir, N. M. Kassim, M. A. Abdulla, and W. A. Yehye, "Hepatoprotective role of ethanolic extract of Vitex negundo in thioacetamide-induced liver fibrosis in male rats," EvidenceBased Complementary and Alternative Medicine, vol. 2013, Article ID 739850, 9 pages, 2013.

[2] Z. A. Amin, M. Bilgen, M. A. Alshawsh, H. M. Ali, A. H. A. Hadi, and M. A. Abdulla, "Protective role of Phyllanthus niruri extract against thioacetamide-induced liver cirrhosis in rat model," Evidence-Based Complementary and Alternative Medicine, vol. 2012, Article ID 241583, 9 pages, 2012. 


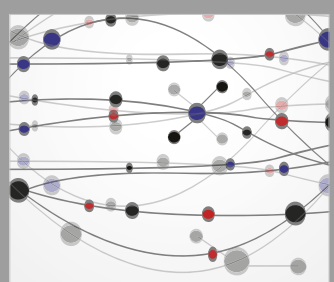

The Scientific World Journal
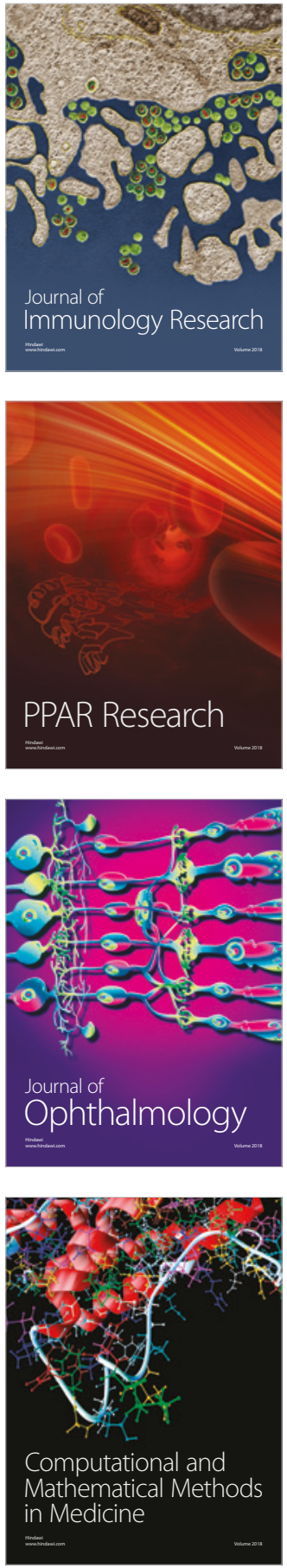

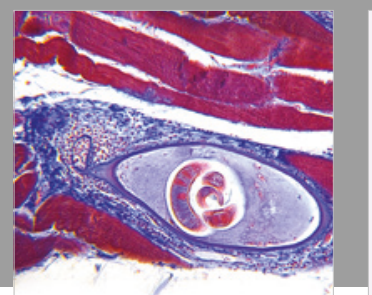

Gastroenterology Research and Practice

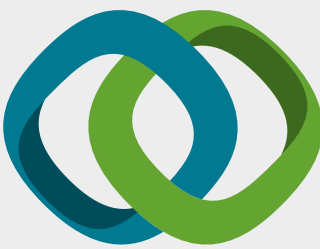

\section{Hindawi}

Submit your manuscripts at

www.hindawi.com
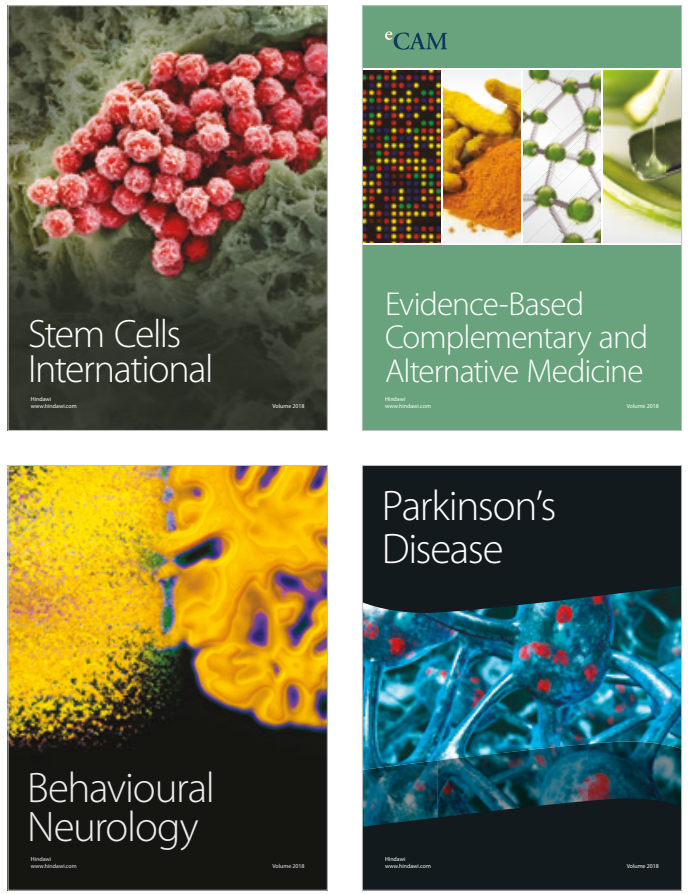

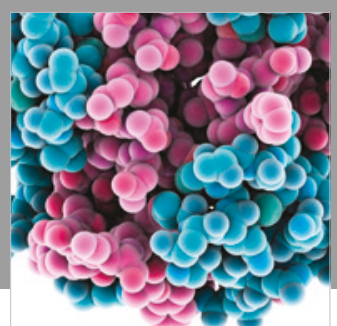

ournal of

Diabetes Research

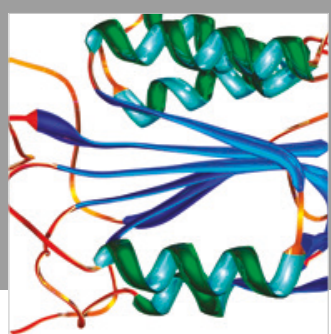

Disease Markers
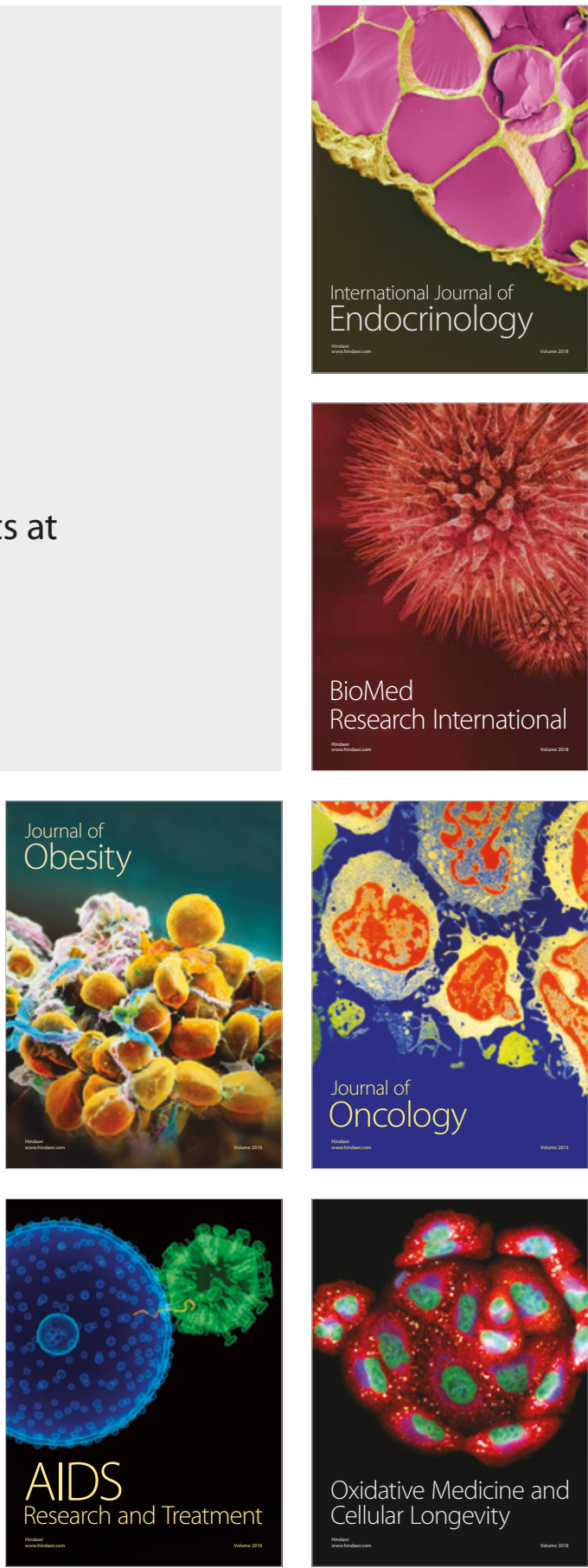